\title{
Factor II, VII, IX and X concentrations in patients receiving long term warfarin
}

\author{
BEVERLEY PAUL, A OXLEY, K BRIGHAM, T COX, ${ }^{*}$ P J HAMILTON \\ From the Department of Haematology, Royal Victoria Infirmary, and the *Department of Statistics, University \\ of Newcastle upon Tyne
}

SUMMARY Using standard one stage clotting assays the concentrations of factors II, VII, IX and X were determined in 37 patients stabilised on warfarin for between three months and 17 years. Contrary to popular belief, the concentrations were not equally depressed, with factor $\mathrm{X}$ the lowest, factor II at intermediate value, factors VII and IX the highest. Some $71 \%$ of the variance of the British corrected ratio (BCR) could be accounted for by measurement of the factors assayed. Analysis of this variance showed $91 \%$ of the explained variance attributable to factor II, $7 \%$ to factor VII, $1.6 \%$ to factor IX and $0.4 \%$ to factor X. With the sudden and recent withdrawal of human thromboplastin, investigation of the sensitivities of the animal thromboplastins to changes in vitamin $\mathrm{K}$ dependent factors in orally anticoagulated patients is needed to ensure that the potentially alarming falls in factors II and $\mathrm{X}$ in these patients are being adequately detected.

The doses of warfarin used for anticoagulation are monitored in most clinics by prothrombin time tests. Such an approach is well tested, and although the thromboplastins used in the prothrombin time test may vary from laboratory to laboratory, the introduction of international standards and national quality control schemes has led to considerable confidence in the performance of prothrombin time tests.

Little interest has been shown in recent years as to how concentrations of the different vitamin $\mathrm{K}$ dependent factors in patients anticoagulated with warfarin may affect the prothrombin time test. This is, we suspect, because popular belief presupposes that coagulation factors are equally depressed in patients stabilised on long term oral anticoagulant treatment. This study questions such conventional wisdom and explores the sensitivity of the prothrombin time test, using Manchester human brain comparative thromboplastin to the differing values of the vitamin $\mathbf{K}$ dependent factors II, VII, IX and X. Since this study was performed and submitted for publication human brain thromboplastins have been withdrawn because of fears of possible viral contamination. Our findings, however, remain of interest, raising as they do important questions as to what is happening to coagulation factors in the long term orally anticoagulated patient and how such treatment should be monitored.

Accepted for publication 30 June 1986

\section{Material and methods}

Thirty seven consecutive outpatients who had received warfarin for not less than three months were studied. There were 20 women aged $28-73$ years (mean 60 years) and 17 men aged $28-78$ years (mean 59 years). Eleven had been receiving warfarin for less than one year, while eight had been treated for longer than 10 years. The average dose was $4.5 \mathrm{mg}$ warfarin per day with wide variation between 1 and $9.5 \mathrm{mg}$ per day. Eighteen patients had valvular heart disease (often with prostheses), 10 arterial thrombosis (stroke, myocardial infarction, peripheral vascular disease), and nine had recurrent venous thrombosis or pulmonary embolus, or both.

\section{VENOUS BLOOD}

Venous blood was withdrawn with minimum stasis and put into plastic tubes, mixing nine volumes of blood with one volume of $3 \cdot 13 \%(0 \cdot 129 \mathrm{M})$ trisodium citrate. Samples were centrifuged at $2000 \mathrm{~g}$ for 10 minutes at $4^{\circ} \mathrm{C}$ to produce platelet poor plasma. Plasma was separated and the British corrected ratio determined immediately from aliquots. The remainder of each sample was stored at $-20^{\circ} \mathrm{C}$ for up to two weeks for factor assays, which were performed in batches using manual methods.

BRITISH CORRECTED RATIOS (BCR)

These were derived after the performance of pro- 
thrombin time tests using the method recommended by the suppliers of the Manchester comparative reagent (MCR), which is identical with British comparative thromboplastin (BCT) (National (UK) Reference Laboratory for Anticoagulant Reagents and Control, Withington Hospital, Manchester) using as control pooled plasmas with a prothrombin time of 12 seconds. Although nomenclature in this field is confusing and not always consistent, ${ }^{1}$ it is important to note that in this study it is the BCR and not the international normalised ratio (INR) that is the appropriate measure.

FACTORS II, VII, AND X

These were measured by standard assay procedures ${ }^{2}$ based on the one stage prothrombin test using an acetone rabbit brain thromboplastin (American Hospital Supplies, Didcot, Berkshire) and relevant substrate plasma of human (factors VII, X) or bovine (factor II) origin from the same suppliers.

\section{FACTOR IX VALUES}

These were assayed by a one stage technique based on the activated partial thromboplastin time using cephalin and activator from American Hospital Supplies and a five minute incubation time.

In all factor assays undertaken four dilutions of normal plasma and three dilutions of the test plasma were prepared in Owren's veronal buffer ( $\mathrm{pH} 7$ 7.35); all tests being performed in duplicate and close attention being paid to parallelism. Because of the importance of relating the different factor values in any individual patient's plasma to those found in all the other patients, combined with the absence of the availability of accepted international reference standards for all factor assays, we calculated all results in this study in terms of the stated values of a commercially available reference plasma (lot no R52) (Immuno AG, Vienna). This lyophilised normal reference plasma is prepared from the citrated plasmas of at least $\mathbf{1 0 0}$ donors and is calibrated in terms of the international standards for factor IX, and a fresh plasma pool of at least 100 donors for factors II, VII, and $\mathrm{X}$.
STA TISTICAL ANALYSES

These were performed in standard ways using a preprogrammed desk top calculator for derivation of the mean, standard deviation, and coefficient of variation of the measurements undertaken in the individual patients and the correlation coefficient ( $r$ ) of any two parameters studied. Multivariate regression analysis of the BCR in terms of the values of factors II, VII, IX and $X$ was performed on the University of Newcastle upon Tyne mainframe computer using the programs Minitab release 80.1 (copyright Pennsylvania State University 1980 ) and Minitab $\mathrm{CP} / \mathrm{M}$ ver 1.3 (copyright CLE, Com Ltd, 1982).

\section{Results}

Table 1 shows the findings of this study. The figure shows the consistency in the pattern of the factor values found in each of the 37 patients. Factor X showed the greatest reduction in values in every patient, with considerable falls in factor II also evident, but in general only modest reductions in factors VII and IX from the accepted normal values of $50-200 \%$ were found. Table 2 shows a simple correlation matrix relating the BCR and the factor values of II, VII, IX and $X$. Because of the significant correlation of the BCR with factors II, VII, IX and $X$ and the high intercorrelation of the individual factors, care must be taken in drawing inferences about the relative contributions made by individual factor values to the BCR. Thus the correlation between the BCR and factor IX, for instance, might merely reflect the significant correlations between factor IX and factor II, or factor VII, or both. To resolve this problem multivariate analysis was undertaken. It is possible to derive from our data, by linear regression analysis, the following equation:

$$
\begin{array}{r}
\text { BCR } \times 10=51.6-(0.34 \text { factor II }+0.12 \text { factor VII } \\
+0.11 \text { factor } X+0.01 \text { factor IX })
\end{array}
$$

Such a relation accounts for $71 \%$ of the variance of the results obtained in this study.

Because of the considerable differences in the absolute values of the factors found in these anticoagulated patients, it is impossible to determine from this equation the relative sensitivity of the BCR

Table 1 Findings in 37 patients receiving warfarin for longer than three months

\begin{tabular}{llll}
\hline & Range & Mean (SD) & Coefficient of variation \\
\hline Warfarin (mg) & $1-9 \cdot 5$ & $4 \cdot 6(2 \cdot 1)$ & 46 \\
BCR & $1 \cdot 5-4 \cdot 8$ & $2 \cdot 89(0 \cdot 66)$ & 23 \\
Factor II & $7-54(\%)$ & $35(11)$ & 31 \\
VII & $18-80(\%)$ & $45(14)$ & 31 \\
IX & $23-76(\%)$ & $49(13)$ & 27 \\
\hline
\end{tabular}




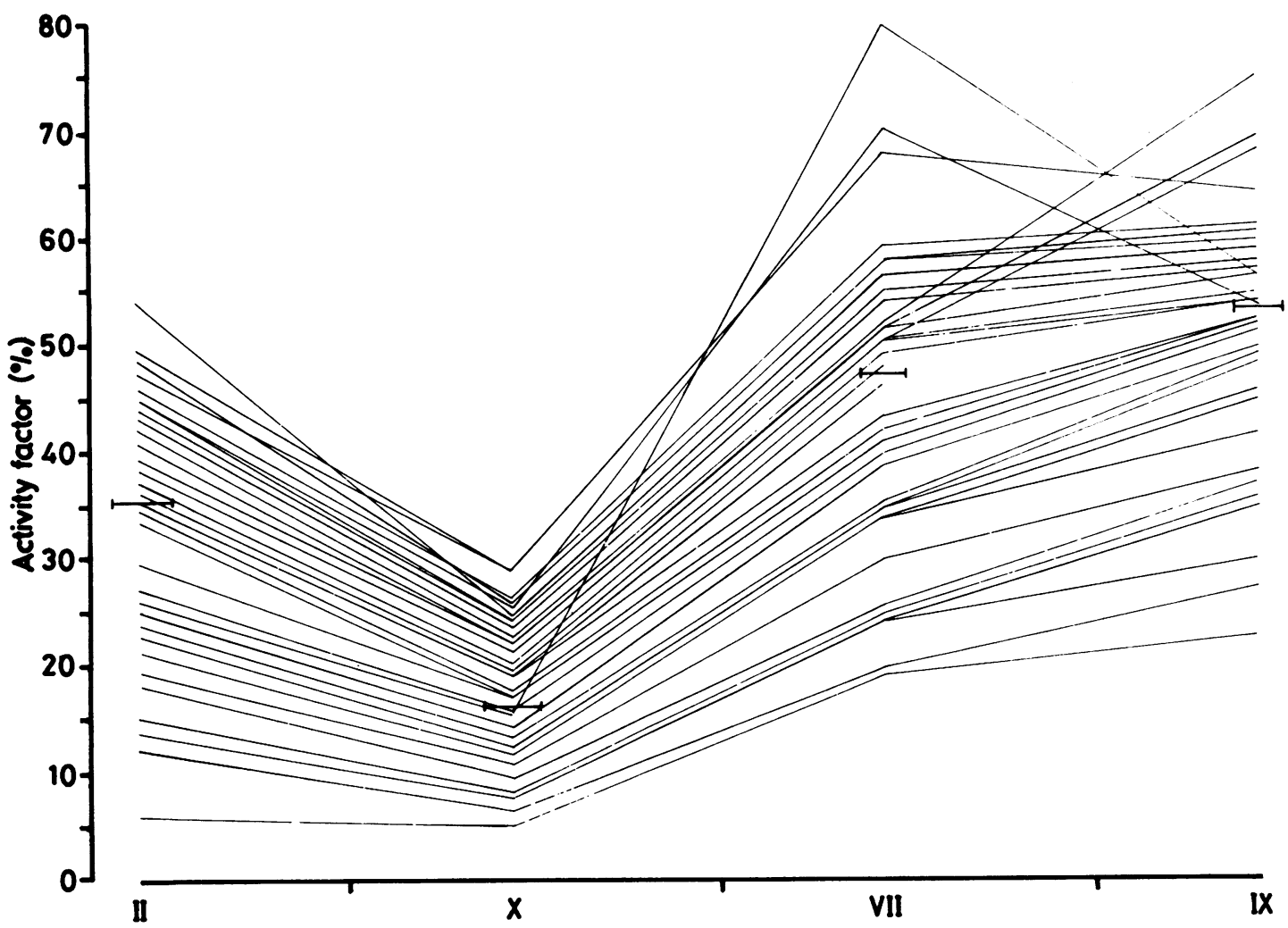

Figure Pattern of factors $I I, V I I, I X$ and $X$ found in individual patients.

to the different factors. By using an analysis of variance and varying the order of variables entered into the analysis, it could be shown that at best factor II accounted for $91 \%$ of the explained variance of the BCR, factor VII, $7 \%$, factor XI, $1.5 \%$, and factor $\mathrm{X}$, $0.4 \%$.

\section{Discussion}

In this study of patients stabilised on long term warfarin anticoagulant treatment a ninefold difference in daily dose of warfarin was controlled by a prothrombin time test, and this was associated with a consistent pattern of differing values of factors II, VII, IX and $\mathrm{X}$ in individual patients. This finding is at odds with opinions expressed in two recent reviews, ${ }^{34}$ which contend that in orally anticoagulated patients concentrations of the vitamin $\mathrm{K}$ dependent coagulation factors fall to about the same values, although at differing rates depending on the half lives of these factors. Such a view was propounded in an essay by Loeliger in $1969,{ }^{5}$ when he suggested that under optimal conditions stable depression of the production rate of the four factors by coumarins was achieved with the attainment of a constant and mutually similar degree of lowered activity to an average value of

Table 2 Correlation matrix of BCR and factors II, VII, IX and X values in 37 patients receiving warfarin for longer than three months

\begin{tabular}{llcccc}
\hline & $B C R$ & $I I$ & $V I I$ & $I X$ & $X$ \\
\hline II & & $-0.84^{* * *}$ & $-0.47^{* *}$ & $-0.52^{* *}$ & $-0.46^{* *}$ \\
VII & $-0.84^{* * *}$ & $0.55^{* * *}$ & $0.55^{* * *}$ & $0.47^{* *}$ & $0.69^{* * *}$ \\
IX & $-0.47^{* *}$ & $0.47^{* *}$ & $0.38^{* *}$ & 0.32 & 0.27 \\
X & $-0.46^{* *}$ & $0.69^{* * *}$ & 0.32 & 0.27 & \\
\end{tabular}

${ }^{* * *} \mathrm{p}<0.001 ;{ }^{* *}$ p 0.01-0.001; *p 0.05-0.01 
around $15-20 \%$ of normal. Inspection of his original work,${ }^{67}$ however, lends scant support for such a view in patients receiving warfarin as anticoagulant. By way of contrast, Winter and Douglas ${ }^{8}$ contend that individual factor values are not depressed to the same extent during anticoagulant treatment, an opinion based on the findings of Owen et al ${ }^{9}$ and amply confirmed in this study: factor $\mathrm{X}$ shows the greatest depression, factor IX the least, with factors II and VII at intermediate levels.

In recent years interest has been shown in the control of oral anticoagulant treatment with individual factor assays exploiting chromogenic amidolytic peptide substrate technology. ${ }^{9-11}$ Presumably, the original rationale for such an approach was based on what we believe is the mistaken view-that is, if all vitamin $\mathrm{K}$ dependent factors fall to about the same values in patients taking warfarin long term then any one of the factors can be chosen and assayed for control of anticoagulant treatment. We suggest that the reason single factor assay may be appropriate for such control is connected with finding similar variations in the parameters of the intensity of anticoagulant treatment studied in these 37 adequately anticoagulated patients. Thus, although the range of the parameters was different (BCR 1.5-4.8, factor II 7-54\%, factor VII $18-80 \%$, factor IX $23-76 \%$, factor X $7-28 \%$ ), their coefficients of variation were very similar $[23,31$, $31,27,31 \%$, respectively]. Thus we suggest that these parameters may, in practice, be interchangeable as measures of intensity of anticoagulant control, as long as the relevant ranges are properly established and verified clinically.

Owren ${ }^{12}$ observed succinctly that, "the goal of anticoagulant therapy is to prevent thrombosis but to preserve haemostasis." Although clinical observation encourages the use and safety of warfarin and other coumarins for satisfactory anticoagulation, the rationale for their use remains uncertain, as does explanation of the occasional unexpected episodes of bleeding that occur despite good control of warfarin anticoagulation with prothrombin time tests. It may not be fortuitous that the different anticoagulants heparin and warfarin exert major effects on factor $\mathbf{X}$ activity and concentration. With regard to the importance of preserving haemostasis in patients receiving warfarin long term, we found that both the values of factors $X$ and II in some patients approached the critical levels of vitamin $\mathrm{K}$ dependent factors below which haemostasis is jeopardised (factor X $8 \%,{ }^{13}$ factor II $9 \% .{ }^{12}$

This study set out to investigate the sensitivity to the different vitamin $K$ dependent factors found in patients taking long term anticoagulants of the measure of anticoagulant control most widely used in Britain at the time (the prothrombin time test expressed as British corrected ratio, or BCR using Manchester human brain thromboplastin). Because this product has been withdrawn, detailed discussion of the multivariate regression analysis of BCR and factors II, VII, IX and $\mathrm{X}$ and the subsequent analysis of variance will not be pursued, as our concern about what the prothrombin time test actually measures will have to be repeated with the animal thromboplastins in current use. Nevertheless, certain findings are worth mentioning. The prothrombin time test is generally considered to be the most sensitive to changes in factor VII and to a lesser extent factor X, but the prothrombin time test is thought to be relatively insensitive to factor II. ${ }^{14}$ In this study factor II accounted for $91 \%$ of the explained variance of BCR, factor VII $7 \%$, and factor $\mathrm{X}$ only $0.4 \%$. The known sensitivities of human brain thromboplastin to factor VII and X deficiencies, when such states occur in isolation, ${ }^{15}$ would seem irrelevant to the changes occurring in the interrelated vitamin $\mathrm{K}$ dependent coagulation factors in patients receiving long term warfarin. Whether the other thromboplastins show similar results is awaited with interest. It will be particularly important to determine whether other prothrombin time tests are sensitive to the low values of factors II and X occurring with warfarin long term because of the critically low values of these factors found in some patients stabilised on long term oral anticoagulant treatment. Thus we note with concern that one of our patients who had been receiving warfarin for 17 years was found to have a BCR of $1 \cdot 6$, factor X of only $12 \%$, while her factor II was $48 \%$, factor VII $70 \%$, and factor IX $66 \%$.

\section{References}

1 Shinton NK. Guidelines on oral anticoagulation. In: Haemostasis and thrombosis task force report. London: British Committee for Standardisation in Haematology, British Society for Haematology.

2 Austen DEG, Rhymes L. Laboratory diagnosis of blood coagulation disorders. In: Biggs R, Rizza CR, eds. Human blood coagulation, haemostasis and thrombosis. 3rd ed. Oxford: Blackwell Scientific Publications, 1984:170-206.

3 Duxbury BMcD. Oral anticoagulant therapy. Hospital Update 1985;11:85-98.

4 Barrowcliffe TW. Standardisation of coagulation tests and assays in quality control. In: Cavill I, ed. Methods in haematology. Vol 4. Edinburgh: Churchill Livingstone, 1982:41-86.

5 Loeliger EA. General considerations on a coumarin induced hypercoagulability and its control. In: Hemker HC, Loeliger EA, Veltkamp JJ, eds. Human Blood coagulation, Boerhaave series. Leiden: University Press, 1969:283-5.

6 Loeliger EA, van der Esch B, Mattern MJ, der Brabander ASA. Behaviour of factors II, VII, IX and X during longterm treatment with coumarin. Thrombosis Diathesis Haemorrhagica 1963;9:74-85.

7 Loeliger EA, Henson A, Mattern MU, Hempker HC. Behaviour of factors II, VII, IX and X in bleeding complications during longterm treatment with coumarin. Thrombosis Diathesis Haemorrhagica 1963;10:278-81. 
8 Winter JH, Douglas AS. Oral anticoagulants. Clin Haematol 1981;10:459-80.

9 Kazmier FJ, Spittell JA, Thompson JJ, Owen CA. Effect of oral anticoagulants on factors VII, IX, X and II. Arch Intern Med 1965;115:667-73.

10 Latallo ZS, Thomson JM, Poller L. An evaluation of chromogenic substrates in the control of anticoagulant therapy. $\mathrm{Br} J$ Haematol 1981;47:307-18.

11 Conkie JA, McCall F, Walker ID, Davidson JF. Evaluation of an automated prothrombin assay using a chromogenic substrate. Haemostasis 1984;14:307-11.

12 Owren PA. Control of anticoagulant therapy. Arch Intern Med 1963;13:166-76.

13 Rizza CR, Matthews JM. Clinical features of clotting factor deficiencies. In: Biggs R, Rizza CR, eds. Human Blood coagulation, haemostasis and thrombosis. 3rd ed. Oxford: Blackwell Scientific Publications, 1984:119-69.

14 Giddings JC. The investigation of hereditary coagulation disorders in blood coagulation and haemostasis. A practical guide. Edinburgh: Churchill Livingstone, 1980.

15 Zucker S, Cathey MH, Cox PJ, Hall EC. Standardisation of laboratory tests for controlling anticoagulant therapy. Am J Clin Pathol 1970;53:348-54.

Requests for reprints to: Dr PJ Hamilton, Department of Haematology, Royal Victoria Infirmary, Newcastle upon Tyne NE1 4LP, England. 